\title{
Infectious endocarditis to an injectable drug user
}

\author{
Adina A. Nanu1,2, Violeta Melinte ${ }^{1,2}$, Simin A. Florescu ${ }^{1,2}$, Adelina Dogaru1, \\ Emanoil Ceausu', ${ }^{1,2}$ Petre I. Calistru ${ }^{1,2}$ \\ 1"Dr. Victor Babes" Clinical Hospital for Infectious and Tropical Diseases, Bucharest, Romania \\ 2"Carol Davila" University of Medicine and Pharmacy, Bucharest, Romania
}

\begin{abstract}
Background. Injecting drug users are a particular category of patients, where infectious pathology, including infectious endocarditis, is much more common. In Romania, in 2016 there were about 21,000 opioid users (www.ana.gov.ro). Besides opioid drugs, there are also ethnobotanics and a growing trend of injecting amphetamines.

Material and methods. We present the case of a young drug user diagnosed in our hospital with infected mitral valve infectious endocarditis.

Particularities consist of the type of affected valve and the involvement of a strain of methicillin-sensitive aureus staphylococcus (in a patient with multiple risk factors for infection with multi-resistant strains).

Results. Clinical evolution is with many ups and downs because different complications occur, but after 54 days of hospitasization he is out, then valvular surgery takes place and he returns to our clinic for the antibiotic consolidation treatment.

Conclusions. This is a case of severe infection in an IDU, with many features indicating a posible negative evolution. Proper antibiotic treatment, associated with other complementary medical interventions, conducted to the favorable uotcome of this young patient.
\end{abstract}

Keywords: infectious endocarditis, injecting drug users, mitral valve,

methicilin-sensitive Staphylococcus aureus

\section{BACKGROUND}

Although with a relatively low incidence (3-10 cases/100,000) (1), infectious endocarditis has a significant impact through frequent complications: congestive heart failure, emboli, mycotic aneurysms, renal dysfunction, abscesses. Approx. 75\% of patients with infectious endocarditis present preexisting cardiac structural conditions (2).

Injecting drug users are a particular category of patients, where infectious pathology, including infectious endocarditis, is much more common.

The main drugs with parenteral administration are opioids. Of these, heroin is most commonly used, but there are also buprenorphine, methadone, fentanyl, codeine, morphine, tramadol, oxycodone. In recent years, we are talking about new psychoactive substances (ethnobotanic). There is also a growing trend of injecting amphetamines, but the prevalence is still low in Romania.

Worldwide, about 12 million people use injectables, of which 1.6 million have HIV infection and 6.1 million HCV infection (2013). There are 1, 3 million active consumers (15-64 years old) in Europe. Nearly $1 / 4$ of the adult population in the EU consumed illegal substances at some point in life (most commonly cannabis). Consumption varies considerably between countries: $1 / 3$ of adults in 
Denmark, France, UK and less than $1 / 10$ adults in Romania, Bulgaria, Turkey(3).

In Romania, in 2016 there were about 21,000 opioid users (80.6\% men, 50.5\% aged 25-34). Prevalence of disease surveillance among consumers admitted for treatment in 2016 was $11.6 \% \mathrm{HIV}$, 4.4\% HBV, 34.3\% HCV (3).

Although Romania is not part of countries with a high prevalence of drug abuse, the upward trend in other countries indicates a similar possibility in our country.

Romania is a trafficking area for illegal drugs (according to the National Anti-drug Agency), so purchasing them can become easier. Measures to combat this behavior, measures to prevent infections caused by injection are not yet well established, so the number of drug users will probably increase in the future and the incidence of drug-related infections such as infectious endocarditis.

Also, an important observation is that the study of consumers admitted for treatment reveals a growing average age. Between 2006 and 2014, the average age of people who have been treated for treatment services has increased by 5 years. At the same time, the mean age of deaths from injecting drug use increased from 33 to 37 years (3).

This category of patients has many features: unfavorable social conditions, malnutrition, poor hygiene, increased nasal and cutaneous colonization with potentially pathogenic infectious agents. Also, behaviors such as injection in areas with increased bacterial load (like inguinal area) predispose to enteral floral infections. Previous hospitalizations, old drug dependence with iv administration, nonprescription antibiotic use predispose to colonization / infection with MRSA. Saliva contamination of the needle or injection site, breaking the capsules into the oral cavity prior to injection, leads to polymicrobial infections with microorganisms from the oral flora (oral streptococci, anaerobic bacteria).

Staphylococcus aureus is the most common cause of bacterial infections among intravenous drug users. Also, infectious endocarditis secondary to bacteraemia with Staphylococcus aureus was more frequently seen in this category compared to the general population, but the literature data supports a better prognosis of intravenous drug users compared to those affected without this risk factor. This difference is not fully understood, but is generally explained by host factors: lower age, lack of preexisting severe heart disease, involvement of right cavities of the heart (4).

\section{CASE REPORT}

D.I., a 30-year-old male, is taken by the ambulance to the emergency room due to high fever $\left(39^{\circ} \mathrm{C}\right)$ with a 3-4 day onset, general condition alteration, generalized myalgia, productive cough. $\mathrm{He}$ is known for chronic hepatitis with $\mathrm{HCV}$ and has a septic episode with cutaneous primary site and pulmonary and meningeal determinations (4 months ago). It is worth mentioning that he is an intravenous drug user for 17 years (heroin, ethnobotanic).

Clinical examination revealed altered general condition, multiple scars, $\mathrm{RR}=25$ breaths $/$ minute, productive cough, bilateral bronchial rales, left pleural rubbing, tachycardia $(115 \mathrm{bpm})$, the edge of the liver 2 centimeters below the ribs, time and space oriented, with no signs of meningeal irritation, very asthenic.

Laboratory samples reveal leukocytosis $(17,000 /$ dl) with neutrophilia $(16,100 / \mathrm{dl})$, mild anemia $(10,1$ $\mathrm{g} / \mathrm{dl})$, moderate thrombocytopenia $(79,000 / \mathrm{dl})$, hyposemia $(126 \mathrm{mmol} / \mathrm{l})$, inflammatory syndrome $(\mathrm{CRP}=16.5 \mathrm{mg} / \mathrm{dl})$, hepatoprivial syndrome (IP = $66 \%, \mathrm{INR}=1.32$, TQ $=16 \mathrm{~s})$, intense positive procalcitonin. Blood is collected for cultures.

Pulmonary X-ray reveals peribronchial alveolar process on the right side.

Stage diagnosis is established, sepsis with presumtive pulmonary primary site, and broad-spectrum empirical antibiotic treatment is initiated: Vancomycin $1 \mathrm{~g} / 12 \mathrm{~h}$ and Meropenem $1 \mathrm{~g} / 8 \mathrm{~h}$.

On the second day of admission, transesophageal echocardiography is performed, which reveals vegetation in the mitral valve $(1.7 \mathrm{~cm})$ and severe mitral regurgitation. Aortic valve with normal appearance, no vegetation, no regurgitation. LV cavity with normal limit dimensions. At the time of examination, without systolic dysfunction, with no clinical phenomenon of heart failure.

Awaiting the results of blood cultures, empirical treatment continues, in order to cover a wide range of bacteria potentially causing the endocardial and pulmonary infection.

After 4 days, 2 of the 3 sets of hemocultures reveal the methicillin-sensitive Staphylococcus aureus strain. 
Thus, according to the modified Duke criteria, the diagnosis of infectious endothelial mitral valve endocarditis with methicillin-sensitive golden staphylococci is determined and the antibiotic treatment is disassembled, replacing with Oxacillin 12 $\mathrm{g}$ /day and Ciprofloxacin $200 \mathrm{mg} / 12$ hours. As it develops an allergic reaction to oxacillin, administration is stopped. The scheme followed will be with Meropenem and Ciprofloxacin. Subsequently, the patient claims dyspnea and abdominal pain while taking Meropenem, so replacing the antibiotic with Vancomycin $1 \mathrm{~g} / 12 \mathrm{~h}$, then in doses adjusted according to the serum residue.

On the radiographic pulmonary image performed 7 days after admission, there is no evidence of the typical pneumonia, but suspicion of hematogenous septic dissemination is raised. After a few days, the clinical examination and then the radiographic image reveal bilateral pleural effusions, so the cardiovascular surgery service is used, where the patient is punctured and $800 \mathrm{ml}$ of serocitrin fluid is evacuated, with favorable post-procedural evolution.

Regular clinical and echocardiographic assessments reveal the persistence of giant vegetation in the mitral valve, with severe regurgitation and a high risk of complications.

After about one month of hospitalization, because of clinical deterioration, pulmonary $\mathrm{x}$-ray is again performed, which reveals a new lung infection process. Under these circumstances, it is added to Vancomycin and Meropenem, Moxifloxacin 400 $\mathrm{mg}$ /day for one week.

According to the therapeutic protocol of infectious endocarditis, the patient is consulted in bucomaxilo-facial surgery, establishing the necessity of rehabilitation of infectious dental outbreaks at a moment after discharge from our department.

At the end of the 6 weeks of antibiotic treatment, the patient presents leukopenia with neutropenia (adverse reaction to long-term treatment with Meropenem), which is why it is decided to delay the discharge and initiate treatment with granulocyte stimulating factors, dexamethasone $16 \mathrm{mg} /$ day, Levofloxacin $500 \mathrm{mg} /$ day and Fluconazole $300 \mathrm{mg} /$ day. Successive, reactive leukocytosis occurs but with spontaneous remission.

After 54 days of hospitalization, it is discharged with the following indications: continuation of the prescribed cardiologic treatment, dental dentist re- habilitation in buco-maxilo-facial surgery clinic (with antibiotic prophylaxis) and addressing in cardiac surgery to assess the opportunity of the surgical cure, under the condition of definitive iv drug withdrawal.

Subsequently, the patient is subjected to the surgery of valvular prosthesis, which takes place without complications. A very important step in the management of infectious endocarditis is the microbiological examination of the excised piece, which dictates the subsequent therapeutic attitude. In our patient's case, the piece was sterile. He returns to our clinic where antibiotic consolidation treatment is instituted with Ceftriaxone and Gentamicin for 2 weeks. Associated, he receives anticoagulant, beta blocker, diuretic and conversion enzyme inhibitor. Evolution is favorable, patient keeps afebril and good overall. He is out with the recommendation of cardiological surveillance.

\section{DISCUSSIONS}

So we are talking about a typical case of severe infectious disease in an iv drug user, but with some notable peculiarities. Both among iv drug users and among the general population, the most frequently incriminated microorganism in the production of infectious endocarditis is Staphylococcus aureus. Peripheral septic embolization (lung, brain, spleen, kidney) is common and affects more than $40 \%$ of patients. These extravascular disorders may provide clues for early diagnosis, especially for IV drug users (4).

The evolution of the presented case was favorable, which corresponds to the data from the literature, which sustain a mortality of only $2-6 \%$ of the infectious endocarditis among the iv drug addicts. This is directly related to the fact that the tricuspid valve is most commonly affected in this population and the infection responds to antibiotic treatment in most cases. In our case, the vegetation was located in the mitral valve. When it affects the mitral or aortic valve, the course of Staphylococcus aureus endocarditis is often fulminant, with generalized metastatic infection leading to death in about $40 \%$ of cases (2).

\section{CONCLUSIONS}

Recent history of hospitalizations and long-lasting iv drug dependence are risk factors for methi- 
cillin resistant Staphylococcus aureus. Lack of infection with a resistant strain probably contributed to favorable progression. Also, the fact that the patient was young influenced the prognostic.

An important feature of the case is hospitalization for sepsis with a cutaneous starting point, 4 months prior to the infective endocarditis episode. In the context of sepsis, there were pulmonary and meningeal determinations, with apparently com-

\section{REFERENCES}

1. Mohamed Sadaka, Eman ELSharkawy, Manal Soliman, Amina Nour EL-Din, Mohamed Ayman Abd EL-Hay. Study of infective endocarditis in Alexandria main uiversity hospitals. The Egyptian Heart Journal, Volume 65, Issue 4, December 2013, Pages 307-317.

2. H. Asgeirsson, A. Thalme, M. Kristjansson, $O$. Weiland. Incidence and outcome of Staphylococcus aureus endocarditis-a 10-year single centre northern European experience. Clinical Mycrobiology and Infection, 2015, Volume 21, Number 8.

3. www.ana.gov.ro. [Online] plete remission at the end of treatment, clinical, paraclinical and radiological parameters being normal. No transesophageal echocardiography was performed, so grafting bacteria and initiating vegetation at the valvular level, if it existed at that time, can not be proven. There remains a question about the link between the two infectious episodes.

Conflict of interest: none declared Financial support: none declared
4. Eeva Ruotsalainen, Minna Karden-Lilja, Pentti Kuusela, Jaana Vuopio-Varkila, Anni Virolainen-Julkunen, Seppo Sarna, Ville Valtonen, Asko Jarvinen. Methicillin-sensitive Staphylococcus aureus bacteraemia and endocarditis among injection drug users and nonaddicts. Journal of infection. Journal of Infection, 2008, Volume56, Issue number4, Pages 249-256.

5. Thomas M. Bashore, Christopher Cabell, Vance Fowler, Jr. Update on Infective Endocarditis. Curr Probl Cardiol. 2006 Apr;31(4):274352. 